\title{
Outcome of Differentiated Thyroid Carcinoma at Two Centres in Kuantan, Pahang
}

Nurul Fatihah Mohd Fuad

Department of Internal Medicine, International Islamic University Malaysia.

Introduction: Differentiated thyroid carcinoma (DTC) is the commonest thyroid malignancy. Several studies had shown that the outcome of DTC depend on the patient's age at time of diagnosis, tumor characteristics and initial treatment. The aim of this study was to describe the outcome of DTC and its associated factors among patients diagnosed at Hospital Tengku Ampuan Afzan (HTAA) and International Islamic University Malaysia Medical Center (IIUMMC) Kuantan, Pahang. Materials and method: A retrospective cohort study was carried out among all DTC patients in HTAA and IIUMMC from July 2018 to September 2018. Case report form was used for data collection and it consists of four sections that include sociodemography, disease characteristics, treatment modalities and outcome. Descriptive statistics was used to describe the types of DTC, sociodemographic characteristics, disease characteristics, treatment modalities and the outcome, while chi-square test was used to explore the factors that were associated with the outcome. Result: In DTC, majority were diagnosed with papillary carcinoma (93.5\%) than medullary carcinoma (6.5\%). Three-quarter of the patients had residual disease at 6 months and the disease persistent in more than half of the patients at 12 months. At two-years follow-up, most of the patients (56.5\%) were in disease-free state followed by persistent $(26.1 \%)$ and residual disease $(17.4 \%)$. However, there were no significant factors that affect the outcome of the patients. Conclusion: The two-year outcome post-treatment in this study revealed most of the patients were in remission. 